\title{
CONVERGENCE OF PERTURBED ALLEN-CAHN EQUATIONS TO FORCED MEAN CURVATURE FLOW
}

\author{
LUCA MUGNAI AND MATTHIAS RÖGER
}

\begin{abstract}
We study perturbations of the Allen-Cahn equation and prove the convergence to forced mean curvature flow in the sharp interface limit. We allow for perturbations that are square-integrable with respect to the diffuse surface area measure. We give a suitable generalized formulation for forced mean curvature flow and apply previous results for the Allen-Cahn action functional. Finally we discuss some applications.
\end{abstract}

\section{INTRODUCTION}

In this paper we study perturbed Allen-Cahn equations of the form

$$
\begin{aligned}
\varepsilon \partial_{t} u_{\varepsilon} & =\varepsilon \Delta u_{\varepsilon}-\frac{1}{\varepsilon} W^{\prime}\left(u_{\varepsilon}\right)+g_{\varepsilon} \quad \text { in } \Omega_{T}, \\
u_{\varepsilon}(0, \cdot) & =u_{\varepsilon}^{0} \quad \text { in } \Omega \\
\nabla u_{\varepsilon} \cdot \nu_{\Omega} & =0 \quad \text { on }(0, T) \times \partial \Omega,
\end{aligned}
$$

where the spatial domain $\Omega$ is given by an open bounded set in $\mathbb{R}^{n}$ with Lipschitz boundary, $(0, T)$ is a fixed time intervall, $\Omega_{T}:=(0, T) \times \Omega$, and $W$ is the standard quartic double-well potential

$$
W(r)=\frac{1}{4}\left(1-r^{2}\right)^{2} .
$$

We are interested in the asymptotics of (1.1) in the sharp interface limit $\varepsilon \rightarrow 0$ for forcing terms $g_{\varepsilon}$ that satisfy

$$
\sup _{\varepsilon>0} \int_{0}^{T} \int_{\Omega} \frac{1}{\varepsilon} g_{\varepsilon}(t, x)^{2} d x d t=: \Lambda<\infty .
$$

Perturbations of this type arise for example in models for diffusion-induced grain boundary motions [9], in models for phase transitions [7], 41], [39], and in image processing [6].

If $g_{\varepsilon}=0$ then (1.1) reduces to the standard Allen-Cahn equation. It is well known that in this case the sharp interface limit is given by the evolution of phase boundaries by mean curvature flow [2, 19, 27]. Our goal is to prove that solutions of the perturbed equation (1.1) converge to motion by forced mean curvature flow,

$$
v=H+g \text {. }
$$

Date: June 16, 2021.

2000 Mathematics Subject Classification. Primary 53C44; Secondary 35K55, 49Q15.

Key words and phrases. Allen-Cahn equation, sharp interface limits, motion by mean curvature. 
Here $v$ describes the velocity vector of an evolution of phase boundaries $\left(\Gamma_{t}\right)_{t \in(0, T)}$, $H(t, \cdot)$ denotes the mean curvature vector of $\Gamma_{t}$, and $g$ is an appropriate limit of $g_{\varepsilon} \nabla u_{\varepsilon}$.

Since the limit evolution in general allows for the formation of singularities in finite time it is necessary to consider suitable generalized formulations of (1.5). In the analysis of mean curvature flow different techniques have been sucessfully applied, in particular viscosity solutions [2, 12, 13, 19, 20], De Giorgi's barriers method [4, 5, 10, 18], and geometric measure theory formulations. We follow here the latter approach and use in particular many ideas from the work of Brakke [8] and Ilmanen [27] on mean curvature flow and the convergence of the Allen-Cahn equation, respectively. To avoid problems with cancellations of phase boundaries we consider not only the evolution of the phases but also the evolution of certain energy measures. In the case of a smooth limit evolution and 'nicely behaving' approximations these measures coincide with the surface area measures associated with the phase boundaries, but in general they may be supported on additional hidden boundaries or may carry a higher mulitplicity. Generalizing hypersurfaces in this way is in the spirit of the theory of (integral) varifolds, which allows to give a meaning to geometric quantities such as mean curvature and second fundamental form, and which provides good compactness properties. In the context of phase transition problems this technique has been successfully applied to a couple of different problems [11, 39, 26, 40, 36, 35, 37.

Our main result is the convergence of solutions to (1.1) to an $L^{2}$-flow of energy measures that move by forced mean curvature flow. The concept of $L^{2}$-flows was develloped in [35] and describes an evolution of integral varifolds with square integrable weak mean curvature and square integrable generalized velocity. We verify the evolution law (1.5) in a pointwise formulation almost everywhere with respect to the energy measures. For a precise formulation of our main result see Section 3.

One benefit of our approach is that we do not use a comparison principle neither for the perturbed Allen-Cahn equation nor for the forced mean curvature flow. This makes our technique quite flexible compared to viscosity solution approaches or to the use of maximum principles to prove the non-positivity of the discrepancy measures, as pursued in [27]. Compared to previous results on forced mean curvature flow [2, 3, 10] and on the convergence of perturbed Allen-Cahn equations our results are more general in the regularity that is required for the forcing term. We do only need that the forcing term is (uniformly) $L^{2}$-integrable with respect to the (diffuse) surface energy measures. On the other hand our proof is limited to space dimensions $n=2,3$ and our formulation of the limit equation is weaker.

This paper borrows many ideas from our analysis of the Allen-Cahn action functional [35], which is defined for any smooth function $u: \Omega_{T} \rightarrow \mathbb{R}$ by

$$
\mathcal{S}_{\varepsilon}(u):=\int_{0}^{T} \int_{\Omega}\left(\sqrt{\varepsilon} \partial_{t} u+\frac{1}{\sqrt{\varepsilon}}\left(-\varepsilon \Delta u+\frac{1}{\varepsilon} W^{\prime}(u)\right)\right)^{2} d x d t .
$$

The functional $\mathcal{S}_{\varepsilon}$ is connected to the small noise limit of the probability of rare events in the stochastically perturbed Allen-Cahn equation [30]. The assumption (1.4) on $g_{\varepsilon}$ yields a uniform bound on the action for solutions $\left(u_{\varepsilon}\right)_{\varepsilon>0}$ of (1.1). By [35] this implies the convergence of diffuse surface area measures associated to $u_{\varepsilon}$ 
to an $L^{2}$-flow in the limit $\varepsilon \rightarrow 0$. In this paper we discuss the convergence of the evolution laws and present some applications. In particular, we prove the convergence of diffuse approximations to the Mullins-Sekerka problem with kinetic undercooling in dimensions $n=2,3$, which improves earlier results by Soner [39].

An important ingredient to derive the compactness of action-bounded sequences and solutions of (1.1) is stated in a (modified) conjecture of De Giorgi [15]: Considering

$$
\begin{aligned}
E_{\varepsilon}(u) & :=\int_{\Omega}\left(\frac{\varepsilon}{2}|\nabla u|^{2}+\frac{W(u)}{\varepsilon}\right) d x, \\
\mathcal{W}_{\varepsilon}(u) & :=\int_{\Omega} \frac{1}{\varepsilon}\left(-\varepsilon \Delta u+\frac{1}{\varepsilon} W^{\prime}(u)\right)^{2} d x
\end{aligned}
$$

the sum $E_{\varepsilon}+\mathcal{W}_{\varepsilon}$ Gamma-converges, up to a constant factor $c_{0}$, to the sum of the Perimeter functional $\mathcal{P}$ and the Willmore functional $\mathcal{W}$,

$$
E_{\varepsilon}+\mathcal{W}_{\varepsilon} \rightarrow c_{0} \mathcal{P}+c_{0} \mathcal{W}, \quad \mathcal{W}(u)=\int_{\Gamma} H^{2} d \mathcal{H}^{n-1},
$$

where $\Gamma$ denotes the phase boundary $\partial^{*}\{u=1\} \cap \Omega$ and where

$$
c_{0}:=\int_{-1}^{1} \sqrt{2 W(s)} d s .
$$

This statement was proved in space dimensions $n=2,3$ by Röger and Schätzle [36] and provides a diffuse version of Allard's compactness theorem for integral varifolds (in the special case of a uniform $L^{2}$ bound on the mean curvature and $n=2,3$ ). In particular we avoid the use of a diffuse version of Huisken's monotonicity formula [24] to derive the rectifiability of the limiting energy measures, as it was done in [27].

\section{2. $L^{2}$-FLOWS AND DIFFUSE SURFACE AREA MEASURES}

In this section we state our weak formulation for evolutions of mean curvature flow type. For basic notions from geometric measure theory we refer to [1, 38].

Notation 2.1. A (general) varifold on $\Omega$ is a Radon measure on the Grassmannian $G^{n-1}(\Omega)$, i.e. the euclidean product of $\Omega$ with the space of unoriented $(n-1)$ planes in $\mathbb{R}^{n}$. A Radon measure $\mu$ on $\Omega$ is $(n-1)$-integer rectifiable if in $\mu$-almost all points $x \in \Omega$ the $(n-1)$-dimensional (measure theoretical) tangent plane $T_{x} \mu$ exists and if $\mu$-almost everywhere the $(n-1)$-dimensional density $\theta^{n-1}(\mu, \cdot)$ is integer-valued. A varifold $V$ on $\Omega$ is $(n-1)$-integer rectifiable if there exists an $(n-1)$-integer rectifiable Radon measure $\mu$ on $\Omega$ such that

$$
\int_{G^{n-1}(\Omega)} \zeta(x, S) d V(x, S)=\int_{\Omega} \zeta\left(x, T_{x} \mu\right) d \mu(x)
$$

for all $\zeta \in C_{c}^{0}\left(G^{n-1}(\Omega)\right)$. This gives a one-to-one correspondence between $(n-1)$ integer rectifiable varifolds and $(n-1)$-integer rectifiable Radon measure on $\Omega$. In this paper we will identify the corresponding objects and use the term integral varifold. 
The first variation $\delta \mu$ of an integral varifold $\mu$ in direction of a vector field $\eta \in$ $C_{c}^{1}\left(\Omega, \mathbb{R}^{n}\right)$ is defined by

$$
\delta \mu(\eta):=\int_{\Omega} \operatorname{div}_{T_{x} \mu} \eta(x) d \mu(x),
$$

where $\operatorname{div}_{T_{x} \mu}$ denotes the divergence restricted to the $(n-1)$-plane $T_{x} \mu$. We say that $\mu$ has a weak mean curvature vector $H_{\mu} \in L_{\text {loc }}^{1}\left(\mu, \mathbb{R}^{n}\right)$ if for all $\eta \in C_{c}^{1}\left(\Omega, \mathbb{R}^{n}\right)$ the first variation is given by

$$
\delta \mu(\eta)=-\int_{\Omega} H(x) \cdot \eta(x) d \mu(x) .
$$

For a family of measures $\left(\mu^{t}\right)_{t \in(0, T)}$ we denote by $\mathcal{L}^{1} \otimes \mu^{t}$ the product measure defined by

$$
\left(\mathcal{L}^{1} \otimes \mu^{t}\right)(\eta):=\int_{0}^{T} \mu^{t}(\eta(t, \cdot)) d t
$$

for any $\eta \in C_{c}^{0}\left(\Omega_{T}\right)$.

We next recall the definition and basic properties of $L^{2}$-flows [35], which describe evolutions of integral varifolds with square integrable mean curvature vector and square integrable generalized velocity.

Definition 2.2. Let $\left(\mu^{t}\right)_{t \in(0, T)}$ be any family of integral varifolds such that $\mu:=$ $\mathcal{L}^{1} \otimes \mu^{t}$ defines a Radon measure on $\Omega_{T}$ and such that $\mu^{t}$ has a weak mean curvature $H(t, \cdot) \in L^{2}\left(\mu^{t}, \mathbb{R}^{n}\right)$ for almost all $t \in(0, T)$.

If there exists a positive constant $C$ and a vector field $v \in L^{2}\left(\mu, \mathbb{R}^{n}\right)$ such that

$$
\begin{aligned}
& v(t, x) \perp T_{x} \mu^{t} \quad \text { for } \mu \text {-almost all }(t, x) \in \Omega_{T}, \\
& \left|\int_{0}^{T} \int_{\Omega}\left(\partial_{t} \eta+\nabla \eta \cdot v\right) d \mu^{t} d t\right| \leq C\|\eta\|_{C^{0}\left(\Omega_{T}\right)}
\end{aligned}
$$

for all $\eta \in C_{c}^{1}((0, T) \times \Omega)$, then we call the evolution $\left(\mu^{t}\right)_{t \in(0, T)}$ an $L^{2}$-flow. A function $v \in L^{2}\left(\mu, \mathbb{R}^{n}\right)$ satisfying (2.1), 2.2) is called a generalized velocity vector.

Remark 2.3. This definition is based on the observation that for a smooth evolution $\left(M_{t}\right)_{t \in(0, T)}$ with square-integrable mean curvature $H(t, \cdot)$ and square-integrable normal velocity vector $V(t, \cdot)$

$$
\begin{aligned}
& \frac{d}{d t} \int_{M_{t}} \zeta(t, x) d \mathcal{H}^{n-1}(x)-\int_{M_{t}} \partial_{t} \zeta(t, x) d \mathcal{H}^{n-1}(x)-\int_{M_{t}} \nabla \zeta(t, x) \cdot V(t, x) d \mathcal{H}^{n-1}(x) \\
= & \int_{M_{t}} H(t, x) \cdot V(t, x) \zeta(t, x) d \mathcal{H}^{n-1}(x) .
\end{aligned}
$$

Integrating this equality in time and using Hölders inequality on the right-hand side implies 2.2.

Any generalized velocity is (in a set of good points) uniquely determined by the evolution $\left(\mu^{t}\right)_{t \in(0, T)}$. In particular, in the case that $\left(\mu^{t}\right)_{t \in(0, T)}$ describes a smooth evolution of smooth hypersurfaces the generalized velocity coincides with the classical velocity of hypersurfaces. 
Proposition 2.4. [35, Proposition 3.3] Let $\left(\mu^{t}\right)_{t \in(0, T)}$ be an $L^{2}$-flow and set $\mu:=$ $\mathcal{L}^{1} \otimes \mu^{t}$. Let $v \in L^{2}\left(\mu, \mathbb{R}^{n}\right)$ be a generalized velocity field in the sense of Definition 2.2. Then

$$
\left(\begin{array}{c}
1 \\
v\left(t_{0}, x_{0}\right)
\end{array}\right) \in T_{\left(t_{0}, x_{0}\right)} \mu
$$

holds in $\mu$-almost all points $\left(t_{0}, x_{0}\right) \in \Omega_{T}$ where the tangential plane of $\mu$ exists. The evolution $\left(\mu^{t}\right)_{t \in(0, T)}$ uniquely determines $v$ in all points $\left(t_{0}, x_{0}\right) \in \Omega_{T}$ where both tangential planes $T_{\left(t_{0}, x_{0}\right)} \mu$ and $T_{x_{0}} \mu^{t_{0}}$ exist.

We next define on the level of phase field approximation diffuse surface area measures.

Definition 2.5. For $\varepsilon>0, t \in(0, T)$ define a Radon measures $\mu_{\varepsilon}^{t}$ on $\Omega$,

$$
\mu_{\varepsilon}^{t}:=\left(\frac{\varepsilon}{2}\left|\nabla u_{\varepsilon}\right|^{2}(t, \cdot)+\frac{1}{\varepsilon} W\left(u_{\varepsilon}(t, \cdot)\right)\right) \mathcal{L}^{n},
$$

and for $\varepsilon>0$ Radon measures $\mu_{\varepsilon}$ on $\Omega_{T}$,

$$
\mu_{\varepsilon}:=\left(\frac{\varepsilon}{2}\left|\nabla u_{\varepsilon}\right|^{2}+\frac{1}{\varepsilon} W\left(u_{\varepsilon}\right)\right) \mathcal{L}^{n+1} .
$$

We will show, that the surface area measures $\mu_{\varepsilon}^{t}$ converge in the limit $\varepsilon \rightarrow 0$ to an $L^{2}$-flow.

To the diffuse surface energy measures $\mu_{\varepsilon}^{t}$ we associate a normal direction $\nu_{\varepsilon}(t, \cdot)$, varifolds $V_{\varepsilon}^{t}$, and Radon measures $\tilde{\mu}_{\varepsilon}$, respectively, defined by

$$
\begin{aligned}
\nu_{\varepsilon} & := \begin{cases}\frac{\nabla u_{\varepsilon}}{\left|\nabla u_{\varepsilon}\right|} & \text { if }\left|\nabla u_{\varepsilon}\right|>0, \\
\vec{e}_{1} & \text { else, }\end{cases} \\
V_{\varepsilon}^{t}(\eta) & :=\int_{\Omega} \eta\left(x, \nu_{\varepsilon}(t, x)^{\perp}\right) d \mu_{\varepsilon}^{t}(x) \quad \text { for } \eta \in C_{c}^{0}\left(\Omega \times G_{n, n-1}\right), \\
\tilde{\mu}_{\varepsilon} & :=\varepsilon\left|\nabla u_{\varepsilon}\right|^{2} \mathcal{L}^{n+1} .
\end{aligned}
$$

\section{Main Results}

Theorem 3.1. Let $n=2,3$ and let $\left(u_{\varepsilon}\right)_{\varepsilon>0},\left(g_{\varepsilon}\right)_{\varepsilon>0}$, and $\left(u_{\varepsilon}^{0}\right)_{\varepsilon>0}$ be given such that (1.1)-(1.4) holds, and such that

$$
\sup _{\varepsilon>0} \mu_{\varepsilon}^{0}(\Omega)=: \Lambda_{0}<+\infty .
$$

Then there exists a subsequence $\varepsilon \rightarrow 0$, a phase indicator function $u \in B V\left(\Omega_{T}\right) \cap$ $L^{\infty}\left(0, T ; B V(\Omega ;\{-1,1\})\right.$, an $L^{2}$-flow $\left(c_{0}^{-1} \mu^{t}\right)_{t \in(0, T)}$, and a vector-field $g \in L^{2}\left(\mu ; \mathbb{R}^{n}\right)$ such that the following properties hold:

(1) Convergence of phase fields

$$
\begin{aligned}
u_{\varepsilon} & \rightarrow u & & \text { in } L^{1}\left(\Omega_{T}\right), \\
u_{\varepsilon}(t, \cdot) & \rightarrow u(t, \cdot) & & \text { in } L^{1}(\Omega) \text { for all } t \in[0, T] .
\end{aligned}
$$

Moreover, $u \in C^{0, \frac{1}{2}}\left([0, T], L^{1}(\Omega)\right)$. 
(2) Convergence of diffuse surface area measures

$$
\begin{array}{ll}
\mu_{\varepsilon} & \rightarrow \mu \\
\mu_{\varepsilon}^{t} & \rightarrow \mu^{t} \quad \text { as Radon-measures on } \Omega_{T}, \\
\mu^{t} \geq \frac{c_{0}}{2}|\nabla u(t, \cdot)| . &
\end{array}
$$

(3) Convergence of force fields

$$
\lim _{\varepsilon \rightarrow 0} \int_{\Omega_{T}}-\eta \cdot \nabla u_{\varepsilon} g_{\varepsilon} d x d t=\int_{\Omega_{T}} \eta \cdot g d \mu
$$

holds for all $\eta \in C_{c}^{0}\left(\Omega_{T}\right)$.

(4) Motion law.

$$
v=H+g
$$

holds $\mu$-almost everywhere, where $H(t, \cdot)$ denotes the weak mean curvature of $\mu^{t}$ and where $v$ denotes the generalized velocity of $\left(\mu^{t}\right)_{t \in(0, T)}$ in the sense of Definition 2.2.

Remark 3.2. Our formulation of forced mean curvature flow can be understood as a generalized formulation of forced mean curvature flow for phase boundaries. In particular, $H, v$, and $g$ restricted to the phase interface $\partial^{*}\{u=1\}$ are a property of $u$ and do not depend on $\mu$. In fact, by [35, Proposition 4.5] we know that $V:=v \cdot \frac{\nabla u}{|\nabla u|}$ belongs to $L^{1}(|\nabla u|)$ and that for every $\eta \in C_{c}^{1}\left(\Omega_{T}\right)$

$$
\int_{0}^{T} \int_{\Omega} V(t, \cdot) \eta(t, \cdot) d|\nabla u(t, \cdot)| d t=-\int_{0}^{T} \int_{\Omega} u(t, x) \partial_{t} \eta(t, x) d x d t
$$

holds. By (2.3) and the rectifiability of $\partial^{*}\{u=1\}$ we conclude that $V$ is $\mathcal{H}^{n}$-almost everywhere on $\partial^{*}\{u=1\}$ uniquely determined by $u$. Moreover, by [31] we have that $H(t, \cdot)$ restricted to $\partial^{*}\{u(t, \cdot)=1\}$ is $\mathcal{H}^{n-1}$-almost everywhere uniquely determined by $u(t, \cdot)$. Therefore $(3.8)$ shows that also $g$ restricted to $\partial^{*}\{u=1\}$ is a property of $u$.

Remark 3.3. Our main results hold also if $\Omega$ is unbounded, with the only change that the limiting phase field $u$ does not belong to $L^{1}\left(\Omega_{T}\right)$ but for all $D \Subset \Omega$ to $L^{1}((0, T) \times D)$ and that the convergence in $(3.2),(3.3)$ is for all $D \Subset \Omega$ in $L^{1}((0, T) \times$ $D)$ and $L^{1}(D)$, respectively. To obtain the corresponding compactness properties, in Proposition 4.2 we use in the case that $\Omega$ is bounded the Modica-Mortola result [33] on the Gamma convergence of the diffuse area. If $\Omega$ is unbounded one uses the Gamma convergence with respect to the $L_{l o c}^{1}$ topology [14] instead.

\section{Proof of Theorem 3.1}

At several instances we will pass to subsequences $\varepsilon \rightarrow 0$ without relabelling. Many arguments are from [35]; to make the paper self-consistent we give in any case at least a sketch of proof. 
4.1. Uniform estimates. By our assumption (1.4) we have a uniform bound on the action functional for the sequence $\left(u_{\varepsilon}\right)_{\varepsilon>0}$, i.e.

$$
\mathcal{S}_{\varepsilon}\left(u_{\varepsilon}\right)=\int_{\Omega_{T}}\left(\sqrt{\varepsilon} \partial_{t} u+\frac{1}{\sqrt{\varepsilon}}\left(-\varepsilon \Delta u+\frac{1}{\varepsilon} W^{\prime}(u)\right)\right)^{2} d x d t=\int_{\Omega_{T}} \frac{1}{\varepsilon} g_{\varepsilon}^{2} d x d t \leq \Lambda .
$$

In particular all results from [35] apply. We next prove uniform bounds for the surface area and for diffuse analog of the velocity and mean curvature. We denote by $w_{\varepsilon}$ the chemical potential, that is the $L^{2}$-gradient of the diffuse surface energy,

$$
w_{\varepsilon}:=-\varepsilon \Delta u_{\varepsilon}+\frac{1}{\varepsilon} W^{\prime}\left(u_{\varepsilon}\right) .
$$

From 1.3), 1.4 we deduce that for any $0 \leq t_{0} \leq T$

$$
\begin{aligned}
\Lambda & \geq \int_{0}^{t_{0}} \int_{\Omega} \frac{1}{\varepsilon}\left(\varepsilon \partial_{t} u_{\varepsilon}+w_{\varepsilon}\right)^{2} d x d t \\
& =\int_{0}^{t_{0}} \int_{\Omega}\left(\varepsilon\left(\partial_{t} u_{\varepsilon}\right)^{2}+\frac{1}{\varepsilon} w_{\varepsilon}^{2}\right) d x d t+2 E_{\varepsilon}\left(u_{\varepsilon}\left(t_{0}, \cdot\right)\right)-2 E_{\varepsilon}\left(u_{\varepsilon}(0, \cdot)\right),
\end{aligned}
$$

which, by (1.4) and (3.1), yields that

$$
\begin{gathered}
\int_{\Omega_{T}}\left(\varepsilon\left(\partial_{t} u_{\varepsilon}\right)^{2}+\frac{1}{\varepsilon} w_{\varepsilon}^{2}\right) d x d t \leq \Lambda+2 \Lambda_{0}, \\
\max _{0 \leq t \leq T} E_{\varepsilon}\left(u_{\varepsilon}(t, \cdot)\right) \leq \frac{1}{2} \Lambda+\Lambda_{0} .
\end{gathered}
$$

We next derive an estimate on the change of the diffuse surface area measures in time.

Lemma 4.1. There exists $C=C\left(\Lambda, \Lambda_{0}\right)$ such that for all $\psi \in C^{1}(\bar{\Omega})$

$$
\int_{0}^{T}\left|\partial_{t} \mu_{\varepsilon}^{t}(\psi)\right| d t \leq C\|\psi\|_{C^{1}(\bar{\Omega})}
$$

Proof. Using (1.3) we compute that

$$
\begin{aligned}
2 \partial_{t} \mu_{\varepsilon}^{t}(\psi)= & \int_{\Omega} \frac{1}{\varepsilon} g_{\varepsilon}(t, x)^{2} \psi(x) d x-\int_{\Omega}\left(\varepsilon\left(\partial_{t} u_{\varepsilon}\right)^{2}+\frac{1}{\varepsilon} w_{\varepsilon}^{2}\right)(t, x) \psi(x) d x \\
& -2 \int_{\Omega} \varepsilon \nabla \psi(x) \cdot \partial_{t} u_{\varepsilon}(t, x) \nabla u_{\varepsilon}(t, x) d x
\end{aligned}
$$

By 4.1), 4.2 we obtain

$$
\begin{aligned}
\left|2 \int_{\Omega_{T}} \varepsilon \nabla \psi \cdot \partial_{t} u_{\varepsilon} \nabla u_{\varepsilon} d x d t\right| & \leq \int_{\Omega_{T}}|\nabla \psi|\left(\varepsilon\left(\partial_{t} u_{\varepsilon}\right)^{2}+\varepsilon\left|\nabla u_{\varepsilon}\right|^{2}\right) d x d t \\
& \leq C\left(\Lambda, \Lambda_{0}, T\right)\|\nabla \psi\|_{C^{0}(\bar{\Omega})}
\end{aligned}
$$

and deduce from (1.4), (4.1), (4.4), (4.5) that

$$
\int_{0}^{T}\left|\partial_{t} \mu_{\varepsilon}^{t}(\psi)\right| d t \leq C\left(\Lambda, \Lambda_{0}, T\right)\left(\|\psi\|_{C^{0}(\bar{\Omega})}+\|\nabla \psi\|_{C^{0}(\bar{\Omega})}\right)
$$

which proves 4.3 . 


\subsection{Convergence of phase fields and diffuse surface area measures.}

Proposition 4.2. There exists a subsequence $\varepsilon \rightarrow 0$ and a phase indicator function $u \in B V\left(\Omega_{T},\{-1,1\}\right) \cap L^{\infty}(0, T ; B V(\Omega))$ such that (3.2), 3.3) hold. Moreover $u \in$ $C^{0,1 / 2}\left(0, T ; L^{1}(\Omega)\right)$.

Proof. By (4.1), 4.2 we can apply the compactness and lower bound from Modica and Mortola [33, 32] in the time-space domain and obtain the existence of $u \in B V\left(\Omega_{T},\{-1,1\}\right)$ such that $(3.2)$ holds for a subsequence $\varepsilon \rightarrow 0$ and such that $u_{\varepsilon}(t, \cdot) \rightarrow u(t, \cdot)$ for almost all $t \in(0, T)$. Next we apply again [33, 32] and we obtain from 4.2 that $u(t, \cdot) \in B V(\Omega)$ with uniformly bounded $B V$-norm. Moreover, for the function

$$
G(r):=\int_{0}^{r} \sqrt{2 W(s)} d s
$$

and almost all $t \in(0, T)$ we have $G\left(u_{\varepsilon}(t, \cdot)\right) \rightarrow \frac{c_{0}}{2} u(t, \cdot)$ and hence for almost all $t_{1}, t_{2}$

$$
\begin{aligned}
\frac{c_{0}}{2} \int_{\Omega}\left|u\left(t_{1}, x\right)-u\left(t_{2}, x\right)\right| d x & =\lim _{\varepsilon \rightarrow 0} \int_{\Omega}\left|G\left(u_{\varepsilon}\left(t_{1}, x\right)\right)-G\left(u_{\varepsilon}\left(t_{2}, x\right)\right)\right| d x \\
& =\int_{\Omega}\left|\int_{t_{1}}^{t_{2}} \partial_{\tau} G\left(u_{\varepsilon}(\tau, x)\right) d \tau\right| d x \\
& =\int_{\Omega}\left|\int_{t_{1}}^{t_{2}} \partial_{\tau} u_{\varepsilon}(\tau, x) \sqrt{2 W\left(u_{\varepsilon}(\tau, x)\right)} d \tau\right| d x \\
& \leq\left(\int_{\Omega_{T}} \varepsilon\left(\partial_{\tau} u_{\varepsilon}(\tau, x)\right)^{2} d x d \tau\right)^{1 / 2}\left|\int_{t_{1}}^{t_{2}} \int \frac{2}{\varepsilon} W\left(u_{\varepsilon}(t, x)\right) d x d t\right|^{1 / 2} \\
& \leq \sqrt{\left|t_{1}-t_{2}\right|} C\left(\Lambda, \Lambda_{0}, T\right),
\end{aligned}
$$

where we have used (4.1), 4.2). This shows that $u$ can be extended to $u \in$ $C^{0,1 / 2}\left([0, T], L^{1}(\Omega)\right)$. Then we also obtain $u_{\varepsilon}(t, \cdot) \rightarrow u(t, \cdot)$ for all $t \in(0, T)$.

We next prove the convergence of the surface area measures $\mu_{\varepsilon}$ and $\mu_{\varepsilon}^{t}$ for almost all times.

Proposition 4.3. There exists a subsequence $\varepsilon \rightarrow 0$ and Radon measures $\mu^{t}, t \in$ $[0, T]$, such that 3.5, (3.6) hold, such that

$$
\mu_{\varepsilon} \rightarrow \mu \quad \text { as Radon measures on } \Omega_{T}, \quad \mu=\mathcal{L}^{1} \otimes \mu^{t},
$$

and such that for all $\psi \in C^{1}(\bar{\Omega})$ the function

$$
t \mapsto \mu^{t}(\psi) \quad \text { is of bounded variation in }(0, T) \text {. }
$$

Proof. [35, Proposition 4.2] By (4.2) we see that $\mu_{\varepsilon}$ is uniformly bounded. Therefore we can select a subsequence $\varepsilon \rightarrow 0$ such that $\mu_{\varepsilon} \rightarrow \mu$ for a Radon-measures $\mu$ on $\Omega_{T}$. Choose next a countable family $\left(\psi_{i}\right)_{i \in \mathbb{N}} \subset C^{1}(\bar{\Omega})$ which is dense in $C^{0}(\bar{\Omega})$. By 
Lemma 4.1 and a diagonal-sequence argument there exists a subsequence $\varepsilon \rightarrow 0$ such that for all $i \in \mathbb{N}$ and almost all $t \in(0, T)$

$$
\begin{gathered}
m_{i}(t):=\lim _{\varepsilon \rightarrow 0} \mu_{\varepsilon}^{t}\left(\psi_{i}\right) \text { exists and } \\
t \mapsto m_{i}(t) \text { is of bounded variation on }(0, T) .
\end{gathered}
$$

Next it is possible to extend (4.9) to the co-countable subset of $[0, T]$ where none of the functions $m_{i}$ has a jump. In a second step we can pass to a subsequence such that (4.9) holds for all $t \in[0, T]$. Taking now an arbitrary $t \in[0, T]$ by 4.2 there exists a subsequence $\varepsilon \rightarrow 0$ such that

$$
\mu^{t}:=\lim _{\varepsilon \rightarrow 0} \mu_{\varepsilon}^{t} \quad \text { exists. }
$$

Hence $\mu^{t}\left(\psi_{i}\right)=m_{i}(t)$ and since $\left(\psi_{i}\right)_{i \in \mathbb{N}}$ is dense in $C^{0}(\bar{\Omega})$ we can identify all limit points of $\left(\mu_{\varepsilon}^{t}\right)_{\varepsilon>0}$ and obtain 4.11 for the whole sequence above and for all $t \in[0, T]$, which proves 3.5. Moreover, by [32] we have

$$
\lim _{\varepsilon \rightarrow 0} \mu_{\varepsilon}^{t} \geq \limsup _{\varepsilon \rightarrow 0}\left|\nabla G\left(u_{\varepsilon}(t, \cdot)\right)\right| \geq|\nabla G(u(t, \cdot))|=\frac{c_{0}}{2}|\nabla u(t, \cdot)| .
$$

By the Dominated Convergence Theorem we conclude that for any $\zeta \in C^{0}\left(\overline{\Omega_{T}}\right)$

$$
\int_{\Omega_{T}} \zeta d \mu=\lim _{\varepsilon \rightarrow 0} \int_{\Omega_{T}} \zeta d \mu_{\varepsilon}=\lim _{\varepsilon \rightarrow 0} \int_{0}^{T} \int_{\Omega} \zeta(t, x) d \mu_{\varepsilon}^{t}(x) d t=\int_{0}^{T} \int_{\Omega} \zeta(t, x) d \mu^{t}(x) d t,
$$

which implies that $\mu$ decomposes as in 4.7 .

4.3. Integrality of the limit measures. One key result is that the limits $\mu^{t}$ of the diffuse surface area measures are not just Radon measures but geometric objects in the sense of being, up to a constant, integer-rectifiable with a weak mean curvature $H(t) \in L^{2}\left(\mu^{t}\right)$. To derive this property we crucially use a compactness property and lower bound proved in [36].

Proposition 4.4. For almost all $t \in(0, T)$

$$
\begin{aligned}
& \frac{1}{c_{0}} \mu^{t} \text { is an integral }(n-1) \text {-varifold, } \\
& \mu^{t} \text { has weak mean curvature } H(t, \cdot) \in L^{2}\left(\mu^{t}, \mathbb{R}^{n}\right) \text {. }
\end{aligned}
$$

Moreover,

$$
\int_{\Omega_{T}}|H|^{2} d \mu \leq \liminf _{\varepsilon \rightarrow 0} \int_{\Omega_{T}} \frac{1}{\varepsilon} w_{\varepsilon}^{2} d x d t<C\left(\Lambda, \Lambda_{0}\right)
$$

and for the Radon measures $\tilde{\mu}_{\varepsilon}$ defined in 2.8 we obtain that

$$
\tilde{\mu}_{\varepsilon} \rightarrow \mu \quad \text { as Radon measures on } \Omega_{T} \text {. }
$$

Finally we deduce that for all $\eta \in C_{c}^{0}\left(\Omega, \mathbb{R}^{n}\right)$

$$
\lim _{\varepsilon \rightarrow 0} \int_{\Omega_{T}} \eta(t, x) \cdot w_{\varepsilon}(t, x) \nabla u_{\varepsilon}(t, x) d x d t=\int_{\Omega_{T}} \eta(t, x) \cdot H(t, x) d \mu(t, x) .
$$


Proof. By 4.1), 4.2 and Fatou's Lemma

$$
\liminf _{\varepsilon \rightarrow 0}\left(\mu_{\varepsilon}^{t}(\Omega)+\frac{1}{\varepsilon} \int_{\Omega} w_{\varepsilon}^{2}(x, t) d x\right)<+\infty,
$$

holds for $\mathcal{L}^{1}$-a.e. $t \in(0, T)$. For every $t \in(0, T)$ such that 4.15 hold we can apply [36, Theorems 4.1 and 5.1, Proposition 4.9]. Hence there exists a subsequence $\varepsilon_{i} \rightarrow 0(i \rightarrow \infty)$ (that may depend on $t$ ) such that $\mu_{\varepsilon_{i}}^{t}$ converge as Radon measures (by $(3.5)$ the limit is given by $\mu^{t}$ ), and such that

$\frac{1}{c_{0}} \mu^{t}$ is an integral varifold with weak mean curvature $H(t, \cdot) \in L^{2}\left(\mu^{t}\right)$,

$$
\int_{\Omega}|H(t, x)|^{2} d \mu^{t}(x) \leq \liminf _{\varepsilon \rightarrow 0} \frac{1}{\varepsilon} \int_{\Omega} w_{\varepsilon}(t, x)^{2} d x .
$$

Moreover, for any subsequence $\varepsilon_{i} \rightarrow 0(i \rightarrow \infty)$ such that 4.15 is satisfied by [36, Theorem 4.1, Proposition 4.9] we have

$$
\begin{gathered}
V_{\varepsilon_{i}}^{t} \stackrel{i \rightarrow \infty}{\rightarrow} \mu^{t} \quad \text { as varifolds on } \Omega, \\
\frac{\varepsilon_{i}}{2}\left|\nabla u_{\varepsilon_{i}}(t, \cdot)\right|^{2}-\frac{1}{\varepsilon_{i}} W\left(u_{\varepsilon_{i}}(t, \cdot)\right) \rightarrow 0 \quad \text { in } L^{1}(\Omega),
\end{gathered}
$$

and by [36, Proposition 4.10] for any $\psi \in C_{c}^{1}(\Omega)$

$$
\begin{aligned}
\lim _{i \rightarrow \infty} \int_{\Omega} \psi(x) \cdot w_{\varepsilon_{i}}(t, x) \nabla u_{\varepsilon_{i}}(t, x) d x & =-\lim _{i \rightarrow \infty} \delta V_{\varepsilon_{i}}^{t}(\psi) \\
& =-\delta \mu^{t}(\psi)=\int_{\Omega} \psi(x) \cdot H(t, x) d \mu^{t}(x) .
\end{aligned}
$$

By a refined version of Lebesgue's Dominated Convergence Theorem it follows [35, Proposition 6.1] that

$$
\begin{gathered}
\frac{\varepsilon}{2}\left|\nabla u_{\varepsilon}\right|^{2}-\frac{1}{\varepsilon} W\left(u_{\varepsilon}\right) \rightarrow 0 \quad \text { in } L^{1}\left(\Omega_{T}\right), \\
\int_{\Omega_{T}} \eta \cdot w_{\varepsilon} \nabla u_{\varepsilon} d x d t \rightarrow \int_{\Omega_{T}} \psi \cdot H d \mu .
\end{gathered}
$$

In particular we deduce 4.13 from 4.20 and 3.4 since

$$
\lim _{\varepsilon \rightarrow 0} \tilde{\mu}_{\varepsilon}=\lim _{\varepsilon \rightarrow 0}\left(\mu_{\varepsilon}+\left(\frac{\varepsilon}{2}\left|\nabla u_{\varepsilon}\right|^{2}-\frac{1}{\varepsilon} W\left(u_{\varepsilon}\right)\right) \mathcal{L}^{n+1}\right)=\mu .
$$

Finally (4.12) follows from 4.16), Fatous Lemma, and 4.1).

\subsection{Existence of a generalized velocity.}

Lemma 4.5. There exists a function $v \in L^{2}\left(\mu, \mathbb{R}^{n}\right)$ such that

$$
\lim _{\varepsilon \rightarrow 0} \int_{\Omega_{T}}-\eta(t, x) \cdot \varepsilon \partial_{t} u_{\varepsilon}(t, x) \nabla u_{\varepsilon}(t, x) d x d t=\int_{\Omega_{T}} \eta(t, x) \cdot v(t, x) d \mu(t, x)
$$


for all $\eta \in C_{c}^{0}\left(\Omega_{T}, \mathbb{R}^{n}\right)$, and such that

$$
\int_{\Omega_{T}}|v|^{2} d \mu \leq \liminf _{\varepsilon \rightarrow 0} \int_{\Omega_{T}} \varepsilon\left(\partial_{t} u_{\varepsilon}\right)^{2} d x d t
$$

Moreover, $\left(c_{0}^{-1} \mu^{t}\right)_{t \in(0, T)}$ is an $L^{2}$-flow with generalized velocity $v$ in the sense of Definition 2.2.

Proof. We prove the lemma in three steps.

Step 1 . We define approximate velocity vectors $v_{\varepsilon}: \Omega_{T} \rightarrow \mathbb{R}^{n}$ by

$$
v_{\varepsilon}:= \begin{cases}-\frac{\partial_{t} u_{\varepsilon}}{\left|\nabla u_{\varepsilon}\right|} \frac{\nabla u_{\varepsilon}}{\left|\nabla u_{\varepsilon}\right|} & \text { if }\left|\nabla u_{\varepsilon}\right| \neq 0 \\ 0 & \text { otherwise }\end{cases}
$$

and deduce from (4.1) that

$$
\int_{\Omega_{T}}\left|v_{\varepsilon}\right|^{2} d \tilde{\mu}_{\varepsilon} \leq \int_{\Omega_{T}} \varepsilon\left(\partial_{t} u_{\varepsilon}\right)^{2} d x d t \leq \Lambda+2 \Lambda_{0}
$$

Therefore $\left(\tilde{\mu}_{\varepsilon}, v_{\varepsilon}\right)$ is a measure-function pair in the sense of [25]. By (4.13) and [25, Theorem 4.4.2] we deduce that there exists a subsequence $\varepsilon \rightarrow 0$ and a function $v \in L^{2}\left(\mu, \mathbb{R}^{n}\right)$ verifying

$$
-\lim _{\varepsilon \rightarrow 0} \int_{\Omega_{T}} \eta \cdot \varepsilon \partial_{t} u_{\varepsilon} \nabla u_{\varepsilon} d x d t=\lim _{\varepsilon \rightarrow 0} \int_{\Omega_{T}} \eta \cdot v_{\varepsilon} d \tilde{\mu}_{\varepsilon}=\int_{\Omega_{T}} \eta \cdot v d \mu,
$$

for every $\eta \in C_{c}^{0}\left(\Omega_{T}, \mathbb{R}^{n}\right)$, which shows 4.23 .

Step 2. We claim that $\left(c_{0}^{-1} \mu^{t}\right)_{t \in(0, T)}$ is an $L^{2}$-flow with generalized velocity $v$. First we have to verify (2.1). With this aim let

$$
P_{\varepsilon}:=I d-\nu_{\varepsilon} \otimes \nu_{\varepsilon}
$$

Moreover denote by $P(t, x): \mathbb{R}^{n} \rightarrow T_{x} \mu^{t} \subset \mathbb{R}^{n}$ the orthogonal projection onto $T_{x} \mu^{t}$ whenever this tangential plane exists, and set $P(t, x)$ to be the orthogonal projection onto $\vec{e}_{1}^{\perp} \subset \mathbb{R}^{n}$ otherwise. Equation (2.1) follows from the identity

$$
\int_{\Omega_{T}} \eta(t, x) \cdot P(t, x) v(t, x) d \mu(t, x)=\lim _{\varepsilon \rightarrow 0} \int_{\Omega_{T}} \eta(t, x) \cdot P_{\varepsilon}(t, x) v_{\varepsilon}(t, x) d \tilde{\mu}_{\varepsilon}(t, x)=0,
$$

which holds for all $\eta \in C_{c}^{0}\left(\Omega_{T}\right)$. The proof of this statement uses arguments from [34, Proposition 3.2]. By the varifold convergence of $V_{\varepsilon}^{t}$ and a Lebesgue-type argument one first obtains strong convergence (in the sense of [25]) for the measure function pair $\left(\tilde{\mu}_{\varepsilon}, P_{\varepsilon}\right)$. Furthermore, by 4.22 we have weak convergence for the measure function pair $\left(\tilde{\mu}_{\varepsilon}, v_{\varepsilon}\right)$ and (4.26) follows, see [35, Lemma 6.3] for details. 
Step 3. In order to conclude we have to prove that $(2.2)$ holds. By similar calculation as in 4.4 we compute that for any $\eta \in C_{c}^{1}\left(\Omega_{T}\right)$,

$$
\begin{aligned}
2 \partial_{t} \mu_{\varepsilon}^{t}(\eta(t, \cdot))= & \int_{\Omega} \frac{1}{\varepsilon} g_{\varepsilon}(t, x)^{2} \eta(t, x) d x-\int_{\Omega}\left(\varepsilon\left(\partial_{t} u_{\varepsilon}\right)^{2}+\frac{1}{\varepsilon} w_{\varepsilon}^{2}\right)(t, x) \eta(t, x) d x \\
& +2 \int_{\Omega} \partial_{t} \eta(t, x) d \mu_{\varepsilon}^{t}(x)-2 \int_{\Omega} \varepsilon \nabla \eta(t, x) \cdot \partial_{t} u_{\varepsilon}(t, x) \nabla u_{\varepsilon}(t, x) d x .
\end{aligned}
$$

Integrating this equality in time and using $(1.4),(4.1)$ we deduce that

$$
\begin{aligned}
& \left|\int_{\Omega_{T}} \partial_{t} \eta(t, x) d \mu_{\varepsilon}(t, x)-\int_{\Omega_{T}} \varepsilon \nabla \eta(t, x) \cdot \partial_{t} u_{\varepsilon}(t, x) \nabla u_{\varepsilon}(t, x) d x d t\right| \\
\leq & C\left(\Lambda, \Lambda_{0}\right)\|\eta\|_{C^{0}\left(\Omega_{T}\right)} .
\end{aligned}
$$

Due to (3.4) and 4.22) this implies (2.2).

4.5. Convergence to forced mean curvature flow. We are now ready to pass to the limit $\varepsilon \rightarrow 0$ in 1.1 .

Proposition 4.6. There exists a subsequence $\varepsilon \rightarrow 0$ and a function $g \in L^{2}\left(\mu, \mathbb{R}^{n}\right)$ such that

$$
\lim _{\varepsilon \rightarrow 0} \int_{\Omega_{T}}-\eta \cdot \nabla u_{\varepsilon} g_{\varepsilon} d x d t=\int_{\Omega_{T}} \eta \cdot g d \mu
$$

for all $\eta \in C_{c}^{0}\left(\Omega, \mathbb{R}^{n}\right)$ and

$$
\int_{\Omega_{T}}|g|^{2} d \mu \leq \liminf _{\varepsilon \rightarrow 0} \int_{\Omega_{T}} \frac{1}{\varepsilon} g_{\varepsilon}^{2}(x, t) d x d t .
$$

Moreover $\left(\mu^{t}\right)_{t \in(0, T)}$ and $g$ satisfy

$$
\int_{\Omega_{T}} \eta \cdot v d \mu=\int_{\Omega_{T}} \eta \cdot(H+g) d \mu
$$

for all $\eta \in C_{c}^{0}\left(\Omega, \mathbb{R}^{n}\right)$, which implies 3.8 . Finally the energy inequality

$$
\begin{gathered}
-\int_{\Omega_{T}} \partial_{t} \zeta d \mu \leq \int_{\Omega_{T}} v \cdot \nabla \zeta d \mu-\frac{1}{2} \int_{\Omega_{T}}\left(|v|^{2}+|H|^{2}\right) \zeta d \mu \\
+\frac{1}{2}\|\zeta\|_{C^{0}\left(\Omega_{T}\right)} \liminf _{\varepsilon \rightarrow 0} \int_{\Omega_{T}} \frac{1}{\varepsilon} g_{\varepsilon}^{2} d x d t .
\end{gathered}
$$

holds for all $\zeta \in C_{c}^{1}\left(\Omega_{T}\right)$ with $\zeta \geq 0$.

Proof. From (1.4) we deduce that

$$
\vec{g}_{\varepsilon}:=-\frac{g_{\varepsilon}}{\varepsilon\left|\nabla u_{\varepsilon}\right|} \nu_{\varepsilon}
$$

satsifies for all $\varepsilon>0$

$$
\int_{\Omega_{T}}\left|\vec{g}_{\varepsilon}\right|^{2} d \tilde{\mu}_{\varepsilon} \leq \int_{\Omega_{T}} \frac{1}{\varepsilon} g_{\varepsilon}^{2} d x d t \leq \Lambda .
$$


Since $\tilde{\mu}_{\varepsilon} \rightarrow \mu$ by 4.13 we deduce from 25] the existence of a subsequence $\varepsilon \rightarrow 0$ and of $g \in L^{2}(\mu)$ such that (4.30) and

$$
\lim _{\varepsilon \rightarrow 0} \int_{\Omega_{T}} \eta \cdot \vec{g}_{\varepsilon} d \tilde{\mu}_{\varepsilon}=\int_{\Omega_{T}} \eta \cdot g d \mu \quad \text { for all } \eta \in C_{c}^{0}\left(\Omega_{T}\right)
$$

holds. By the definition of $\tilde{\mu}_{\varepsilon}$ and $\vec{g}_{\varepsilon}$ this is equivalent to 4.29. Multiplying (1.1) with $-\eta \cdot \nabla u_{\varepsilon}$ and integrating yields

$$
\int_{\Omega_{T}}-\eta \cdot \varepsilon \partial_{t} u_{\varepsilon} \nabla u_{\varepsilon} d x d t=\int_{\Omega_{T}} \eta \cdot \nabla u_{\varepsilon} w_{\varepsilon}-\eta \cdot g_{\varepsilon} \nabla u_{\varepsilon} d x d t
$$

Using 4.14, 4.22, 4.29) we can pass to the limit $\varepsilon \rightarrow 0$ in this equation and arrive at (4.31).

To prove 4.32 we rewrite 4.27) and integrate in time to obtain

$$
\begin{aligned}
\int_{\Omega_{T}}-\partial_{t} \zeta d \mu_{\varepsilon}= & -\int_{\Omega} \varepsilon \nabla \zeta(t, x) \cdot \partial_{t} u_{\varepsilon}(t, x) \nabla u_{\varepsilon}(t, x) d x d t \\
& -\frac{1}{2} \int_{\Omega_{T}} \zeta\left(\varepsilon\left(\partial_{t} u_{\varepsilon}\right)^{2}+\frac{1}{\varepsilon} w_{\varepsilon}^{2}\right) d x d t+\int_{\Omega_{T}} \frac{1}{2 \varepsilon} g_{\varepsilon}^{2} \zeta d x d t .
\end{aligned}
$$

By (3.4), 4.22), and (4.12), 4.23) we then deduce 4.32).

\section{Applications}

5.1. Small perturbations of the Allen-Cahn equation and motion by mean curvature. For 'small perturbations' of the Allen-Cahn equation in the sense that

$$
\int_{\Omega_{T}} \frac{1}{\varepsilon} g_{\varepsilon}^{2}(t, x) d x d t \rightarrow 0 \quad \text { as } \varepsilon \rightarrow 0
$$

Theorem 3.1 implies that we obtain motion by mean curvature in the limit. This shows a stability of the convergence of the Allen-Cahn equation to mean curvature flow. In fact, we obtain here the convergence to an enhanced motion in the sense of Ilmanen [28].

Proposition 5.1. Assume that (1.1)-(1.3) hold, and that the perturbations $\left(g_{\varepsilon}\right)_{\varepsilon>0}$ satisfy (5.1). Then the conclusions of Theorem 3.1 hold with $g=0$. Moreover, $\left(\mu^{t}\right)_{t \in(0, T)}$ is a Brakke motion. If we in addition assume that the initial data are well-prepared, in the sense that

$$
\lim _{\varepsilon \rightarrow 0} \mu_{\varepsilon}^{0} \rightarrow \frac{c_{0}}{2}|\nabla u(0, \cdot)|,
$$

then the $L^{2}$-flow $\left(\frac{1}{c_{0}} \mu^{t}\right)_{t \in(0, T)}$ together with the current associated to $\partial^{*}\{u=1\}$ is an enhanced motion in the sense of [28] with initial condition $\partial^{*}\{u(0, \cdot)=1\}$.

Proof. By (5.1) we can apply Theorem 3.1 and by 4.30 we obtain that $g=0$. From (4.32) and (3.8) we further conclude that

$$
-\int_{\Omega_{T}} \partial_{t} \zeta d \mu \leq \int_{\Omega_{T}} H \cdot \nabla \zeta d \mu-\int_{\Omega_{T}} \zeta|H|^{2} d \mu
$$


holds for all $\zeta \in C_{c}^{1}\left(\Omega_{T}\right)$ with $\zeta \geq 0$. This is a time-integrated version of Brakkes inequality. Now one derives from (5.3), following Evans and Spruck [21, Theorem 7.1], that $\left(\mu_{t}\right)_{t \in(0, T)}$ is a Brakke motion.

Further, we have proved in [35, Proposition 8.2] that there exists a nonnegative function $p \in L^{2}(\mu)$ such that

$$
\frac{c_{0}}{2}\left|\nabla^{\prime} u\right| \leq p \mu
$$

where $\nabla^{\prime}=\left(\partial_{t}, \nabla_{x}\right)^{T}$ denotes the time-space gradient in $\mathbb{R} \times \mathbb{R}^{n}$. This implies by (4.2) that

$$
\begin{gathered}
\int_{(t, t+\tau) \times \Omega} \frac{c_{0}}{2} d\left|\nabla^{\prime} u\right|(t, x) \\
\leq\|p\|_{L^{2}(\mu)}\left(\int_{t}^{t+\tau} \mu^{s}(\Omega) d s\right)^{1 / 2} \leq \tau^{\frac{1}{2}}\|p\|_{L^{2}(\mu)} C\left(\Lambda, \Lambda_{0}, T\right) .
\end{gathered}
$$

By (3.6), (5.2), (5.4), and since $\left(\mu^{t}\right)_{t \in(0, T)}$ moves by Brakke motion, we can conclude that $\left(\mu^{t}\right)_{t \in(0, T)}$ and $\partial^{*}\{u=1\}$ constitute an enhanced motion with initial condition $\partial^{*}\{u(0, \cdot)=1\}$.

Remark 5.2. For an enhanced motion Ilmanen [28] proves consistency and regularity results. In particular, the initial surface $\partial^{*}\left\{u^{0}=1\right\}$ can be perturbed to one whose evolution is smooth $\mathcal{H}^{n}$-almost everywhere in $\Omega_{T}$.

Remark 5.3. Actually the conclusions of Proposition 4.6 still hold under weaker assumptions on the perturbation $g_{\varepsilon}$, namely it is sufficient that $\left(g_{\varepsilon}\right)_{\varepsilon>0}$ satisfies (1.4) and that (3.7) holds with $g=0$.

5.2. Equation with perturbed double-well potential and a drift term. In this section we consider (1.1) with perturbations of the form

$$
g_{\varepsilon}(t, x)=\varepsilon b_{\varepsilon}(t, x) \cdot \nabla u_{\varepsilon}(t, x)+f_{\varepsilon}(t, x) \sqrt{2 W\left(u_{\varepsilon}(t, x)\right)}
$$

with $b_{\varepsilon}: \Omega_{T} \rightarrow \mathbb{R}^{n}, f_{\varepsilon}: \Omega_{T} \rightarrow \mathbb{R}$ given. Whereas the first term describes a drift, the term $f_{\varepsilon}(t, x) \sqrt{W\left(u_{\varepsilon}(t, x)\right)}$ may arise from a perturbation of the double well potential. Kobayashi [29], for instance, introduced such a term as a thermodynamic driving force in a model for dendritic crystal growth. He proposed a potential of the form

$$
W_{\varepsilon}(r, m)=W(r)+\left(\frac{2}{3}(r+1)^{3}-2(r+1)^{3}\right) m,
$$

with $m=\varepsilon f$, where $f$ may be a function depending on other quantities such as the temperature. This gives

$$
\partial_{r} W_{\varepsilon}(r, m)=W^{\prime}(r)+2\left(r^{2}-1\right) m=W^{\prime}(r)+4 m \sqrt{W(r)} .
$$

and yields in (1.1) a pertubative term

$$
g_{\varepsilon}=4 f \sqrt{W\left(u_{\varepsilon}\right)}
$$

Barles and Soner [3] and Barles, Soner, and Souganidis [2 considered phase field models of Allen-Cahn type with a perturbation of the form (5.5). They proved the 
convergence to forced mean curvature flow in a viscosity solutions formulations under the assumption that $b_{\varepsilon}=b_{\varepsilon}(x)$ and $f_{\varepsilon}=f_{\varepsilon}(t, x)$ are uniformly Lipschitz continuous in time and space.

Our Theorem 3.1 covers this situation under weaker assumptions on the regularity of the forcing term.

Proposition 5.4. Consider (1.1) with a perturbation of the form (5.5) and assume that there exists $\Lambda_{1}>0$ independent of $\varepsilon>0$ such that

$$
\int_{0}^{T} \sup _{x \in \Omega}\left(\left|f_{\varepsilon}(t, x)\right|^{2}+\left|b_{\varepsilon}(t, x)\right|^{2}\right) d t \leq \Lambda_{1} .
$$

Then the conclusions of Theorem 3.1 hold. Moreover, we obtain that the limiting forcing term is given by

$$
g(t, x)=-(P(t, x)-I d) b-f(t, x),
$$

where $P(t, x): \mathbb{R}^{n} \rightarrow T_{x} \mu^{t}$ denotes the orthogonal projection onto the tangential plane $T_{x} \mu^{t}$ of $\mu^{t}$ and $b$ and $f$ are determined by

$$
\begin{aligned}
\int_{\Omega_{T}} b \cdot \eta d \mu & =\lim _{\varepsilon \rightarrow 0} \int_{\Omega_{T}} b_{\varepsilon} \cdot \eta \varepsilon\left|\nabla u_{\varepsilon}\right|^{2} d x d t \\
\int_{\Omega_{T}} f \cdot \eta d \mu & =\lim _{\varepsilon \rightarrow 0} \int_{\Omega_{T}} f_{\varepsilon} \sqrt{2 W\left(u_{\varepsilon}\right)} \nabla u_{\varepsilon} \cdot \eta d x d t .
\end{aligned}
$$

Finally, in the case that $b_{\varepsilon}, f_{\varepsilon}$ are continuous and converge as $\varepsilon \rightarrow 0$ uniformly in $\Omega_{T}$ to $\widehat{b}$ and $\widehat{f}$, then $b=\widehat{b}$ on $\operatorname{supp}(\mu)$ and $f \mu=\frac{c_{0}}{2} \widehat{f} \nabla u$.

Proof. We have to verify that $g_{\varepsilon}$ satisfies 1.4 . We first show a uniform bound on the diffuse surface surface area. With this aim we set as above $w_{\varepsilon}=-\varepsilon \Delta u_{\varepsilon}+\frac{1}{\varepsilon} W^{\prime}\left(u_{\varepsilon}\right)$ and compute

$$
\begin{aligned}
& \frac{d}{d t} \int_{\Omega}\left(\frac{\varepsilon}{2}\left|\nabla u_{\varepsilon}\right|^{2}(t, x)+\frac{1}{\varepsilon} W\left(u_{\varepsilon}(t, x)\right)\right) d x \\
= & \int_{\Omega} w_{\varepsilon}(t, x) \partial_{t} u_{\varepsilon}(t, x) d x \\
= & \int_{\Omega}-\frac{1}{\varepsilon} w_{\varepsilon}^{2}(t, x)+w_{\varepsilon}(t, x)\left(b_{\varepsilon}(t, x) \cdot \nabla u_{\varepsilon}(t, x)+\frac{1}{\varepsilon} f_{\varepsilon}(t, x) \sqrt{W\left(u_{\varepsilon}(t, x)\right)}\right) d x \\
\leq & \int_{\Omega}\left(\frac{\varepsilon}{2}\left|b_{\varepsilon}(t, x)\right|^{2}\left|\nabla u_{\varepsilon}(t, x)\right|^{2}+\frac{1}{\varepsilon} f_{\varepsilon}(t, x)^{2} W\left(u_{\varepsilon}(t, x)\right)\right) d x \\
\leq & \sup _{x \in \Omega}\left(\left|f_{\varepsilon}(t, x)\right|^{2}+\left|b_{\varepsilon}(t, x)\right|^{2}\right) \int_{\Omega}\left(\frac{\varepsilon}{2}\left|\nabla u_{\varepsilon}\right|^{2}(t, x)+\frac{1}{\varepsilon} W\left(u_{\varepsilon}(t, x)\right)\right) d x .
\end{aligned}
$$

Hence Gronwall's inequality and (5.6) imply that

$$
E_{\varepsilon}\left(u_{\varepsilon}(t, \cdot)\right) \leq E_{\varepsilon}\left(u_{\varepsilon}(0, \cdot)\right) e^{\Lambda_{1}} .
$$


Under the assumption (3.1) on the initial data we deduce that

$$
\begin{aligned}
\int_{\Omega_{T}} \frac{1}{\varepsilon} g_{\varepsilon}(t, x)^{2} d x & d t \leq 2 \int_{\Omega_{T}}\left(\varepsilon\left|b_{\varepsilon}(t, x)\right|^{2}\left|\nabla u_{\varepsilon}(t, x)\right|^{2}+\frac{1}{\varepsilon} f_{\varepsilon}(t, x)^{2} W\left(u_{\varepsilon}(t, x)\right)\right) d x d t \\
& \leq 4 \int_{0}^{T} \sup _{x \in \Omega}\left(\left|f_{\varepsilon}(t, x)\right|^{2}+\left|b_{\varepsilon}(t, x)\right|^{2}\right) E_{\varepsilon}\left(u_{\varepsilon}(t, \cdot)\right) d t \\
& \leq 4 \Lambda_{0} e^{\Lambda_{1}} \Lambda_{1}
\end{aligned}
$$

which verifies (1.4). Therefore we obtain (1.5) with $g$ satisfying (3.7). To prove the representation formula (5.7), with $b, f$ as in (5.8), (5.9), we compute

$$
\begin{aligned}
\int_{\Omega_{T}}-\eta \cdot \nabla u_{\varepsilon} g_{\varepsilon} d x d t= & \int_{\Omega_{T}}-\eta \cdot\left(P_{\varepsilon}-I d\right) b_{\varepsilon} d \tilde{\mu}_{\varepsilon} \\
& -\int_{\Omega_{T}} f_{\varepsilon} \sqrt{2 W\left(u_{\varepsilon}\right)} \nabla u_{\varepsilon} \cdot \eta d x d t
\end{aligned}
$$

To characterize the limit of the right-hand side of this equation we first observe that by 4.2 , (5.6) we have

$$
\sup _{\varepsilon>0} \int_{\Omega_{T}}\left|b_{\varepsilon}\right|^{2}+\left|\vec{f}_{\varepsilon}\right|^{2} d \tilde{\mu}_{\varepsilon} \leq 2 \sup _{\varepsilon>0} \int_{0}^{T} \sup _{x \in \Omega}\left(\left|b_{\varepsilon}(t, x)\right|^{2}+f_{\varepsilon}(t, x)^{2}\right) E_{\varepsilon}\left(u_{\varepsilon}(t, \cdot)\right) d t<\infty,
$$

where we defined

$$
\vec{f}_{\varepsilon}:=f_{\varepsilon} \frac{\sqrt{2 W\left(u_{\varepsilon}\right)}}{\varepsilon\left|\nabla u_{\varepsilon}\right|} \nu_{\varepsilon} .
$$

By 4.20 and [25, Theorem 4.4.2] there exist $b, f \in L^{2}(\mu)$ and a subsequence $\varepsilon \rightarrow 0$ such that (5.8) and

$$
\int_{\Omega_{T}} f \cdot \eta d \mu=\lim _{\varepsilon \rightarrow 0} \int_{\Omega_{T}} \vec{f}_{\varepsilon} \cdot \eta d \tilde{\mu}_{\varepsilon}=\lim _{\varepsilon \rightarrow 0} \int_{\Omega_{T}} \eta \cdot f_{\varepsilon} \sqrt{2 W\left(u_{\varepsilon}\right)} \nabla u_{\varepsilon} d x d t
$$

which is (5.9), are satisfied. By varifold convergence and an argument similar to that one used in [34, Proposition 3.2], [35, Lemma 6.3] one obtains that $\left(P_{\varepsilon}, \tilde{\mu}_{\varepsilon}\right)$ converges to $(P, \mu)$ strongly as measure-function pairs. Together with (5.8) this implies that

$$
\lim _{\varepsilon \rightarrow 0} \int_{\Omega_{T}}-\eta \cdot\left(P_{\varepsilon}-I d\right) b_{\varepsilon} d \tilde{\mu}_{\varepsilon}=\int_{\Omega_{T}}-\eta \cdot(P-I d) b d \mu .
$$

By (5.9), (5.12), this proves the characterization of $g$.

In the case that $b_{\varepsilon} \rightarrow \hat{b}$ uniformly in $\Omega_{T}$ we obtain from (5.8) that $b=\hat{b}$ on $\operatorname{supp}(\mu)$. To characterize $f$ in $(5.9)$ we observe that for $G$ as in 4.6

$$
\int_{\Omega_{T}} \eta \cdot f_{\varepsilon} \sqrt{2 W\left(u_{\varepsilon}\right)} \nabla u_{\varepsilon} d x d t=\int_{\Omega_{T}} \eta \cdot f_{\varepsilon} \nabla G\left(u_{\varepsilon}\right) d x d t
$$

By [32] we have that $\nabla G\left(u_{\varepsilon}\right) \rightarrow \frac{c_{0}}{2} \nabla u_{\varepsilon}$ weakly as measures and since $f_{\varepsilon} \rightarrow f$ uniformly we conclude that

$$
\int_{\Omega_{T}} f \cdot \eta d \mu=\frac{c_{0}}{2} \int_{\Omega_{T}} f \eta \cdot \nabla u .
$$


Remark 5.5. By the same arguments we also can allow for pertubations of the form $g_{\varepsilon}=\varepsilon f_{\varepsilon}\left|\nabla u_{\varepsilon}\right|$ as were considered by Benes and Mikula [7] in a model for phase transitions and by Benes, Chalupecký, and Mikula in image processing [6].

\subsection{Application to Mullins-Sekerka problem with kinetic undercooling.}

Here we apply our main Theorem in a situation where the forcing term in the limit is not concentrated on the phase interface but rather given by the trace of a Sobolev function in the ambient space. As a concrete application we prove the convergence of phase field approximations of the Mullins-Sekerka problem with kinetic undercooling. This improves in space dimensions $n=2,3$ an earlier result by Soner [39]. Throughout this section we will assume $\Omega=\mathbb{R}^{n}$. As noticed in Remark 3.3 our main results apply also to this case. Let us consider the Allen-Cahn equation with perturbations $g_{\varepsilon}$ that are given by

$$
g_{\varepsilon}(t, x)=\theta_{\varepsilon}(t, x) \sqrt{2 W\left(u_{\varepsilon}(t, x)\right)}
$$

where we now assume that $\theta_{\varepsilon}(t, \cdot) \in C^{1}(\Omega)$ for all $t \in \mathbb{R}$ and that

$$
\sup _{\varepsilon>0} \int_{\Omega_{T}}\left(\theta_{\varepsilon}^{2}+\left|\nabla \theta_{\varepsilon}\right|^{2}\right) d x d t<\infty
$$

We first show that we can derive from this control of $\theta_{\varepsilon}$ in the bulk that the assumption (1.4), which was necessary to apply Theorem 3.1, is satisfied by $g_{\varepsilon}$.

Proposition 5.6. Let sequences $\left(u_{\varepsilon}\right)_{\varepsilon>0},\left(\theta_{\varepsilon}\right)_{\varepsilon>0}$ be given and define $g_{\varepsilon}$ by (5.14). Assume that (5.15) is satisfied and that $u_{\varepsilon}, g_{\varepsilon}$ are solutions of (1.1)-(1.3). Furthermore let (3.1) hold for the initial data $u_{\varepsilon}^{0}$ and assume that we have a uniform upper bound on the density of the diffuse surface area measures,

$$
\sup _{x \in \mathbb{R}^{n}, R>0} \frac{\mu_{\varepsilon}^{t}\left(B_{R}(x)\right)}{R^{n-1}} \leq K(T), \quad \forall t \in[0, T) .
$$

Then $g_{\varepsilon}$ satisfies 1.4 .

Proof. By [42, Theorem 5.12.4] it follows from (5.16) that for any $\varphi \in C_{c}^{1}\left(\mathbb{R}^{n}\right)$

$$
\left|\int_{\mathbb{R}^{n}} \varphi(x) d \mu(x)\right| \leq K_{n} M(\mu) \int_{\mathbb{R}^{n}}|\nabla \varphi| d x .
$$

For $R>1$ we choose a smooth cut-off function $\varphi_{R} \in C_{c}^{1}\left(\mathbb{R}^{n}\right)$ with $\varphi_{R} \geq 0$ on $\mathbb{R}^{n}$, $\varphi_{R} \equiv 1$ on $B_{R}, \varphi_{R} \equiv 0$ on $B_{2 R}$, and $\left\|\nabla \varphi_{R}\right\|_{L^{\infty}\left(\mathbb{R}^{n}\right)} \leq 1$. We then obtain that

$$
\frac{1}{\varepsilon} \int_{B_{R}} g_{\varepsilon}^{2}(t, x) d x=\int_{B_{R}} \theta_{\varepsilon}^{2}(t, x) \frac{W\left(u_{\varepsilon}(t, x)\right)}{\varepsilon} d x \leq \int_{\mathbb{R}^{n}} \varphi_{R}(x) \theta_{\varepsilon}^{2}(t, x) d \mu_{\varepsilon}^{t}(x) .
$$


Applying (5.17) with $\varphi=\varphi_{R} \theta_{\varepsilon}^{2}(t, \cdot)$ we deduce that the right-hand-side of (5.18) is estimated by

$$
\begin{aligned}
\int_{\mathbb{R}^{n}} \varphi_{R}(x) \theta_{\varepsilon}^{2}(t, x) d \mu_{\varepsilon}^{t}(x) & \leq K_{n} K(T) \int_{\mathbb{R}^{n}}\left|\nabla\left(\varphi_{R} \theta_{\varepsilon}^{2}(t, \cdot)\right)\right| d x \\
& \leq K_{n} K(T) \int_{\mathbb{R}^{n}} \theta_{\varepsilon}^{2}(t, \cdot)\left|\nabla \varphi_{R}\right|+2 \varphi_{R}\left|\theta_{\varepsilon}(t, \cdot) \nabla \theta_{\varepsilon}(t, \cdot)\right| d x \\
& \leq K_{n} K(T) \int_{\mathbb{R}^{n}}\left(2 \theta_{\varepsilon}^{2}(t, \cdot)+\left|\nabla \theta_{\varepsilon}(t, \cdot)\right|^{2}\right) d x .
\end{aligned}
$$

With $R \rightarrow \infty$ we deduce from (5.18), the last inequality, and (5.15) that (1.4) holds.

Next we apply Proposition 5.6 to the phase-fields approximation of the MullinsSekerka problem with kinetic undercooling introduced in [39]. More precisely, let $\left(u_{\varepsilon}, \theta_{\varepsilon}\right)_{\varepsilon>0}$ be the unique, bounded, smooth solutions on $Q:=(0,+\infty) \times \mathbb{R}^{n}$ to the following Cauchy problem

$$
\begin{aligned}
\varepsilon \partial_{t} u_{\varepsilon} & =\varepsilon \Delta u_{\varepsilon}-\frac{W^{\prime}\left(u_{\varepsilon}\right)}{\varepsilon}+\sqrt{2 W\left(u_{\varepsilon}\right)} \theta_{\varepsilon} & & \text { in } Q, \\
\partial_{t} \theta_{\varepsilon} & =\Delta \theta_{\varepsilon}-\sqrt{2 W\left(u_{\varepsilon}\right)} \partial_{t} u_{\varepsilon} & & \text { in } Q, \\
u_{\varepsilon}(0, \cdot) & =u_{\varepsilon}^{0}, \quad \theta_{\varepsilon}(0, \cdot)=\theta_{\varepsilon}^{0}, & & \text { in } \mathbb{R}^{n} .
\end{aligned}
$$

Proposition 5.7. Let $n=2,3$ and let $\left(u_{\varepsilon}, \theta_{\varepsilon}\right)_{\varepsilon>0}$ satisfy (5.19)-(5.21). Assume that the initial data $u_{\varepsilon}^{0}, \theta_{\varepsilon}^{0}$ are well-prepared in the sense of [39, Section 2.4] and that

$$
\begin{gathered}
\sup _{\varepsilon>0}\left\|u_{\varepsilon}^{0}\right\|_{L^{\infty}\left(\mathbb{R}^{n}\right)} \leq 1, \\
\sup _{\varepsilon>0} \int_{\mathbb{R}^{n}}\left(\frac{\varepsilon}{2}\left|\nabla u_{\varepsilon}^{0}\right|^{2}+\frac{W\left(u_{\varepsilon}^{0}\right)}{\varepsilon}+\left(\theta_{\varepsilon}^{0}\right)^{2}\right) d x<C_{1}
\end{gathered}
$$

hold. Then there exists a subsequence $\varepsilon \rightarrow 0$ (not relabelled) and functions

$$
\begin{aligned}
& \theta \in L_{l o c}^{\infty}\left(0, \infty ; L^{2}\left(\mathbb{R}^{n}\right)\right) \cap L_{l o c}^{2}\left(0, \infty ; H^{1,2}\left(\mathbb{R}^{n}\right)\right), \\
& u \in B V_{l o c}(Q) \cap L^{\infty}\left(0, \infty ; B V_{l o c}\left(\mathbb{R}^{n},\{-1,1\}\right),\right.
\end{aligned}
$$

such that

$$
\begin{aligned}
& \theta_{\varepsilon} \rightarrow \theta \quad \text { weakly in } L_{l o c}^{2}\left(0, \infty ; H^{1,2}\left(\mathbb{R}^{n}\right)\right), \\
& \theta_{\varepsilon}(t, \cdot) \rightarrow \theta(t, \cdot) \quad \text { in } L_{l o c}^{2}\left(\mathbb{R}^{n}\right) \text {, for every } t \geq 0 \text {, }
\end{aligned}
$$

and

$$
u_{\varepsilon} \rightarrow u \quad \text { in } L_{\mathrm{loc}}^{p}(Q) \text { for all } 1 \leq p<\infty .
$$

Moreover the conclusion of Theorem 3.1 hold. In particular, there exists a measurable function $\alpha: \partial^{*}\{u=1\} \rightarrow \mathbb{N}$ such that, in the generalized formulation of Theorem 3.1 ,

$$
v=H-\frac{1}{\alpha} \theta \nu \quad \mathcal{H}^{n}-\text { almost everywhere on } \partial^{*}\{u=1\},
$$

where $\nu$ denotes the inner normal of $\{u=1\}$ on $\partial^{*}\{u=1\}$. 
Finally we have for every $\eta \in C_{c}^{\infty}(Q)$ that

$$
-\int_{Q}\left(\partial_{t} \eta+\Delta \eta\right) \theta d x d t=\frac{c_{0}}{2} \int_{Q} u \partial_{t} \eta d x d t
$$

Remark 5.8. An example of well-prepared intial data is given by $\theta_{\varepsilon}^{0}=\theta^{0} \in C_{c}^{2}\left(\mathbb{R}^{n}\right)$, and $u_{\varepsilon}^{0}(x):=\gamma_{\varepsilon}\left(d_{\partial E}(x) / \varepsilon\right)$, where $d_{\partial E}$ denotes the signed distance from the smooth boundary of the open subset $E$ of $\mathbb{R}^{n}$, while $\gamma_{\varepsilon}$ are appropriate approximations of the optimal transition profile $\tanh (\cdot / \sqrt{2})$.

Proof of Proposition 5.7. We show that Proposition 5.6 can be applied. The key estimates have been proved in [39]. Firstly by (5.22) and the maximum principle $\left|u_{\varepsilon}(t, x)\right| \leq 1$ for every $(t, x) \in Q$. Hence (5.14) is satifsfied. Next, solutions of (5.19)-(5.21) satisfy the energy identity

$$
\mu_{\varepsilon}^{t}\left(\mathbb{R}^{d}\right)+\left\|\theta_{\varepsilon}(t, \cdot)\right\|_{L^{2}\left(\mathbb{R}^{n}\right)}^{2}+\int_{0}^{t} \int_{\mathbb{R}^{n}}\left(\varepsilon\left(\partial_{t} u_{\varepsilon}\right)^{2}+\left|\nabla \theta_{\varepsilon}\right|^{2}\right) d x d t=\mu_{\varepsilon}^{0}\left(\mathbb{R}^{d}\right)+\left\|\theta_{\varepsilon}^{0}\right\|_{L^{2}\left(\mathbb{R}^{n}\right)}
$$

and (5.24)-(5.26) follow from (5.29) and [39, Section 2.3, Proposition 3.4]. Finally the crucial estimate (5.16) was shown in [39, Proposition 7.2]. By Remark 3.3 and Proposition 5.6 we now can apply Theorem 3.1. To derive the limiting forcing term $g$ we consider the function $G$ defined in (4.6) and observe that that by (3.7) for all $\eta \in C_{c}^{1}(Q)$

$$
\begin{aligned}
\int_{Q} \eta \cdot g d \mu & =\lim _{\varepsilon \rightarrow 0} \int_{Q}-\eta \cdot \theta_{\varepsilon} \sqrt{2 W\left(u_{\varepsilon}\right)} \nabla u_{\varepsilon} d x d t \\
& =\lim _{\varepsilon \rightarrow 0} \int_{Q}-\eta \cdot \theta_{\varepsilon} \nabla G\left(u_{\varepsilon}\right) d x d t \\
& =\lim _{\varepsilon \rightarrow 0} \int_{Q} G\left(u_{\varepsilon}\right) \nabla \cdot\left(\eta \theta_{\varepsilon}\right) d x d t=\frac{c_{0}}{2} \int_{Q} u \nabla \cdot(\eta \theta) d x d t,
\end{aligned}
$$

where we have used (5.24), 5.26). Now let $\alpha(t, x):=\theta^{n-1}\left(c_{0}^{-1} \mu^{t}, x\right)$ denote the $(n-1)$-dimensional density of $c_{0}^{-1} \mu^{t}$ in $x$. By the integrality of $c_{0}^{-1} \mu^{t}$ we obtain that $\alpha$ is integer valued $\mathcal{H}^{n}$-almost everywhere. From $(5.30)$ we deduce that

$$
g c_{0} \alpha=-c_{0} \theta \nu
$$

$\mathcal{H}^{n}$-almost everywhere on $\partial^{*}\{u=1\}$ and 5.27 follows from 3.8 .

To derive (5.28) we first multiply (5.20) with $\eta \in C_{c}^{1}(Q)$, integrate over $Q$, do some partial integrations, and use that $\sqrt{2 W\left(u_{\varepsilon}\right)} \partial_{t} u_{\varepsilon}=\partial_{t} G\left(u_{\varepsilon}\right)$. This gives

$$
\int_{Q} \partial_{t} \eta \theta_{\varepsilon} d x d t=\int_{Q} \nabla \eta \cdot \nabla \theta_{\varepsilon}-\partial_{t} \eta G\left(u_{\varepsilon}\right) d x d t
$$

and by (5.24), (5.26) we conclude that (5.28) holds.

Remark 5.9. Proposition 5.7 improves the results obtained in 39 for space dimensions $n \leq 3$. Firstly we have shown that $c_{0}^{-1} \mu^{t}$ are for $\mathcal{L}^{1}$-a.e. $t \in(0,+\infty)$ integer rectifiable, which implies by [8, Section 5.8] that the generalized mean curvature vector $H(t, \cdot)$ is $\mu^{t}$-a.e. orthogonal to $T_{x} \mu^{t}$. Secondly we are closer to a pointwise 
formulation of the interface motion law on the phase boundary. The occurrence of an integer factor $\alpha$ in (5.27) is typical in the varifold approach to the convergence in phase field equations, see for example [11] and [40], or [37] for a situation where this problem could be resolved.

5.4. Application to a model for diffusion induced grain boundary motion. As another application we discuss a model for diffusion induced grain boundary motion proposed by Cahn, Fife, and Penrose [9] that was analyzed in a couple of different papers [22, 16, 17, 23]. The model describes the dynamics of two phases of different orientations in a polycrystalline film and of the concentration of certain atoms that diffuse along the grain boundaries from outside into the film. The system is driven by the reduction of surface area of the grain boundary and a driving force that depends on the concentration of the metal. In the model a free surface area of the form

$$
\mathcal{F}_{\varepsilon}\left(u_{\varepsilon}, c_{\varepsilon}\right)=\int_{\Omega} \frac{\varepsilon}{2}\left|\nabla u_{\varepsilon}\right|^{2}+\frac{1}{\varepsilon} W\left(u_{\varepsilon}\right)+\frac{1}{2 \varepsilon} c_{\varepsilon}^{2}+\left(u_{\varepsilon}+1\right) f\left(c_{\varepsilon}\right) d x
$$

is considered, where $u_{\varepsilon}$ is a phase field that describes two different crystal-lattice orientations indicated by the values $u_{\varepsilon}= \pm 1$ and where $c_{\varepsilon}$ denotes the concentration field of atoms. Here $W$ is a double well potential (typically a double obstacle potential) and $f$ is a given globally Lipschitz continuous function with $f(0)=0$ and $f(r) \geq 0$ for $r \in[0,1]$, which is the range of physically meaningful values for $c_{\varepsilon}$. A gradient flow dynamic is assumed, with respect to a scalar product that is of $L^{2}-$ type in the first component and of $H^{-1}$-type with degenerate mobility in the second component,

$$
\left\|\left(v_{1}, v_{2}\right)\right\|_{\left(u_{\varepsilon}, c_{\varepsilon}\right)}^{2}:=\left\|v_{1}\right\|_{L^{2}(\Omega)}^{2}+\int_{\Omega} D\left(u_{\varepsilon}\right)\left|\nabla Z\left(v_{2}\right)\right|^{2}
$$

for a tangent vector $\left(v_{1}, v_{2}\right)$ at $\left(u_{\varepsilon}, c_{\varepsilon}\right)$ and for a suitable degenerate mobility function $D$. Here $z=Z\left(v_{2}\right)$ denotes the solution of

$$
-\nabla\left(D\left(u_{\varepsilon}\right) \nabla z\right)=v_{2}
$$

These choices lead to a system of equations

$$
\begin{aligned}
& \varepsilon \partial_{t} u_{\varepsilon}=\varepsilon \Delta u_{\varepsilon}-\frac{1}{\varepsilon} W^{\prime}\left(u_{\varepsilon}\right)+f\left(c_{\varepsilon}\right), \\
& \varepsilon \partial_{t} c_{\varepsilon}=\nabla \cdot\left(D\left(u_{\varepsilon}\right) \nabla\left(c_{\varepsilon}+\varepsilon\left(u_{\varepsilon}+1\right) f^{\prime}\left(c_{\varepsilon}\right)\right)\right),
\end{aligned}
$$

complemented by initial and boundary conditions,

$$
\begin{aligned}
u_{\varepsilon}(0, \cdot) & =u_{\varepsilon}^{0}, \quad c_{\varepsilon}(0, \cdot)=c_{\varepsilon}^{0} \quad \text { in } \Omega \\
\nabla u_{\varepsilon} \cdot \nu_{\Omega} & =0, \quad D\left(u_{\varepsilon}\right) \nabla c_{\varepsilon} \cdot \nu_{\Omega}=0 \quad \text { on }(0, T) \times \partial \Omega .
\end{aligned}
$$

We choose in the following $W$ to be the standard quartic double well potential, assume that $f$ is linear and consider the convergence as $\varepsilon \rightarrow 0$ of (5.32) only. 
Proposition 5.10. Let a sequence $\left(u_{\varepsilon}, c_{\varepsilon}\right)_{\varepsilon>0}$ of solutions of $(5.32)-(5.35)$ be given, let $f(r)=r$, and assume that the initial data satisfy

$$
\mathcal{F}_{\varepsilon}\left(u_{\varepsilon}^{0}, c_{\varepsilon}^{0}\right) \leq \Lambda_{0}
$$

for all $\varepsilon>0$. Then there exists a subsequence $\varepsilon \rightarrow 0$, a phase indicator function $u \in B V\left(\Omega_{T}\right) \cap L^{\infty}\left(0, T ; B V(\Omega ;\{-1,1\})\right.$, a $L^{2}$-flow $\left(\mu^{t}\right)_{t \in(0, T)}$, and a function $c \in$ $L^{2}\left(\mu ; \mathbb{R}^{n}\right)$ such that the conclusions of Theorem 3.1 hold. In particular we obtain that for all $\eta \in C_{c}^{0}\left(\Omega_{T}\right)$

$$
\lim _{\varepsilon \rightarrow 0} \int_{\Omega_{T}} \eta \cdot \nabla u_{\varepsilon} c_{\varepsilon} d x d t=\int_{\Omega_{T}} \eta \cdot c d \mu
$$

and that

$$
H=v+c
$$

holds $\mu$-almost everywhere.

Proof. Let us firstly notice that for every for every $\varepsilon$ small enough

$$
\frac{1}{2 \varepsilon} W\left(u_{\varepsilon}\right)+\frac{1}{4 \varepsilon} c_{\varepsilon}^{2} \leq 1+\frac{1}{\varepsilon} W\left(u_{\varepsilon}\right)+\frac{1}{2 \varepsilon} c_{\varepsilon}^{2}+c_{\varepsilon}\left(1+u_{\varepsilon}\right)
$$

Since $t \mapsto \mathcal{F}_{\varepsilon}\left(u_{\varepsilon}(t, \cdot), c_{\varepsilon}(t, \cdot)\right)$ is nonincreasing under the gradient flow dynamics we obtain from the above inequality and (5.36) that

$$
\frac{1}{2} E_{\varepsilon}\left(u_{\varepsilon}(t, \cdot)\right)+\frac{1}{4} \int_{\Omega} \frac{1}{\varepsilon} c_{\varepsilon}^{2}(t, x) d x \leq \mathcal{L}^{n}(\Omega)+\mathcal{F}_{\varepsilon}\left(u_{\varepsilon}(t, \cdot), c_{\varepsilon}(t, \cdot)\right) \leq \Lambda_{0}+\mathcal{L}^{n}(\Omega) .
$$

This latter inequality furnishes the uniform bound needed to apply Theorem 3.1, and the conclusions follow.

Remark 5.11. We do not address here the questions of convergence in (5.33) and of the right choice for the mobility function $D$ in the case of a quartic double-well potential. We expect that taking (5.33) into account will improve the convergence of $c_{\varepsilon}$ and in (5.32) and will allow to prove (5.33) for more general Lipschitz functions $f$.

\section{REFERENCES}

[1] W. K. Allard. On the first variation of a varifold. Annals of Mathematics, 95:417-491, 1972.

[2] G. Barles, H. M. Soner, and P. E. Souganidis. Front propagation and phase field theory. SIAM J. Control Optim., 31(2):439-469, 1993.

[3] G. Barles and P. E. Souganidis. A new approach to front propagation problems: theory and applications. Arch. Rational Mech. Anal., 141(3):237-296, 1998.

[4] G. Bellettini and M. Novaga. Minimal barriers for geometric evolutions. J. Differential Equations, 139(1):76-103, 1997.

[5] G. Bellettini and M. Paolini. Some results on minimal barriers in the sense of De Giorgi applied to driven motion by mean curvature. Rend. Accad. Naz. Sci. XL Mem. Mat. Appl. (5), 19:43$67,1995$.

[6] M. Benes, V. Chalupecký, and K. Mikula. Geometrical image segmentation by the allen-cahn equation. Applied Numerical Mathematics, 51(2-3):187-205, Nov 2004.

[7] M. Beneš and K. Mikula. Simulation of anisotropic motion by mean curvature - comparison of phase field and sharp interface approaches. In Proceedings of the Algoritmy'97 Conference on Scientific Computing (Zuberec), volume 67, pages 17-42, 1998. 
[8] K. A. Brakke. The motion of a surface by its mean curvature, volume 20 of Mathematical Notes. Princeton University Press, Princeton, N.J., 1978.

[9] J. Cahn, P. Fife, and O. Penrose. A phase-field model for diffusion-induced grain-boundary motion. Acta Materialia, 45(10):4397-4413, 1997. DOI: 10.1016/S1359-6454(97)00074-8.

[10] A. Chambolle and M. Novaga. Implicit time discretization of the mean curvature flow with a discontinuous forcing term. Interfaces and Free Boundaries, pages 283-300, 2008.

[11] X. Chen. Global asymptotic limit of solutions of the Cahn-Hilliard equation. J. Diff. Geom., 44:262-311, 1996.

[12] Y. G. Chen, Y. Giga, and S. Goto. Uniqueness and existence of viscosity solutions of generalized mean curvature flow equations. J. Diff. Geom. 33, 749-786, 1991.

[13] M. G. Crandall, H. Ishii, and P.-L. Lions. User's guide to viscosity solutions of second order partial differential equations. Bull. Amer. Math. Soc. (N.S.), 27(1):1-67, 1992.

[14] G. Dal Maso and L. Modica. A general theory of variational functionals. In Topics in functional analysis, 1980-81, Quaderni, pages 149-221. Scuola Norm. Sup. Pisa, Pisa, 1981.

[15] E. De Giorgi. Some remarks on $\Gamma$-convergence and least squares method. In Composite media and homogenization theory (Trieste, 1990), volume 5 of Progr. Nonlinear Differential Equations Appl., pages 135-142. Birkhäuser Boston, Boston, MA, 1991.

[16] K. Deckelnick and C. M. Elliott. An existence and uniqueness result for a phase-field model of diffusion-induced grain-boundary motion. Proc. Roy. Soc. Edinburgh Sect. A, 131(6):13231344, 2001.

[17] K. Deckelnick, C. M. Elliott, and V. Styles. Numerical diffusion-induced grain boundary motion. Interfaces Free Bound., 3(4):393-414, 2001.

[18] N. Dirr, S. Luckhaus, and M. Novaga. A stochastic selection principle in case of fattening for curvature flow. Calc. Var. Partial Differential Equations, 13(4):405-425, 2001.

[19] L. C. Evans, H. M. Soner, and P. E. Souganidis. Phase transitions and generalized motion by mean curvature. Comm. Pure Appl. Math., 45(9):1097-1123, 1992.

[20] L. C. Evans and J. Spruck. Motion of level sets by mean curvature. I. J. Diff. Geom. 33, 635-681, 1991.

[21] L. C. Evans and J. Spruck. Motion of level sets by mean curvature. IV. J. Geom. Anal., 5(1):77-114, 1995.

[22] P. C. Fife, J. W. Cahn, and C. M. Elliott. A free-boundary model for diffusion-induced grain boundary motion. Interfaces Free Bound., 3(3):291-336, 2001.

[23] H. Garcke, R. Nürnberg, and V. Styles. Stress- and diffusion-induced interface motion: modelling and numerical simulations. European J. Appl. Math., 18(6):631-657, 2007.

[24] G. Huisken. Asymptotic behavior for singularities of the mean curvature flow. J. Differential Geom., 31(1):285-299, 1990.

[25] J. E. Hutchinson. Second fundamental form for varifolds and the existence of surfaces minimising curvature. Indiana Univ. Math. J., 35:45-71, 1986.

[26] J. E. Hutchinson and Y. Tonegawa. Convergence of phase interfaces in the van der WaalsCahn-Hilliard theory. Calc. Var. Partial Differential Equations, 10(1):49-84, 2000.

[27] T. Ilmanen. Convergence of the Allen-Cahn equation to Brakke's motion by mean curvature. J. Differential Geom., 38(2):417-461, 1993.

[28] T. Ilmanen. Elliptic regularization and partial regularity for motion by mean curvature. Mem. Amer. Math. Soc., 108, 1994.

[29] R. Kobayashi. Modeling and numerical simulations of dendritic crystal growth. Physica D: Nonlinear Phenomena, 63(3-4):410-423, 1993.

[30] R. Kohn, F. Otto, M. G. Reznikoff, and E. Vanden-Eijnden. Action minimization and sharpinterface limits for the stochastic Allen-Cahn equation. Comm. Pure Appl. Math., 60(3):393438, 2007.

[31] U. Menne. Second order rectifiability of integral varifolds of locally bounded first variation, 2008. 
[32] L. Modica. The gradient theory of phase transitions and the minimal interface criterion. Arch. Rational Mech. Anal., 98:357-383, 1987.

[33] L. Modica and S. Mortola. Un esempio di Г-convergenza. Boll. Un. Mat. Ital. B (5), 14(1):285299, 1977.

[34] R. Moser. A generalization of Rellich's theorem and regularity of varifolds minimizing curvature. Preprint 72, Max Planck Institute for Mathematics in the Sciences, Leipzig, 2001.

[35] L. Mugnai and M. Röger. The Allen-Cahn action functional in higher dimensions. Interfaces Free Bound., 10(1):45-78, 2008.

[36] M. Röger and R. Schätzle. On a modified conjecture of De Giorgi. Mathematische Zeitschrift, 254(4):675-714, 2006.

[37] M. Röger and Y. Tonegawa. Convergence of phase-field approximations to the Gibbs-Thomson law. Calc. Var. Partial Differential Equations, 32(1):111-136, 2008.

[38] L. Simon. Lectures on geometric measure theory, volume 3 of Proceedings of the Centre for Mathematical Analysis, Australian National University. Australian National University Centre for Mathematical Analysis, Canberra, 1983.

[39] H. M. Soner. Convergence of the phase-field equations to the Mullins-Sekerka problem with kinetic undercooling. Arch. Rational Mech. Anal., 131(2):139-197, 1995.

[40] Y. Tonegawa. A diffused interface whose chemical potential lies in a Sobolev space. Ann. Sc. Norm. Super. Pisa Cl. Sci. (5), 4(3):487-510, 2005.

[41] S.-L. Wang and et al. Thermodynamically-consistent phase-field models for solidification. Phys. D, 69(1-2):189-200, 1993.

[42] W. Ziemer. Interior and boundary continuity of weak solutions of degenerate parabolic equations. Trans. Amer. Math. Soc., 271:733-748, 1982.

Luca Mugnai, Max Planck Institute for Mathematics in the Sciences, Inselstr. 22, D-04103 LEIPZIG

Matthias Röger, Max Planck Institute for Mathematics in the Sciences, Inselstr. 22, D-04103 LEIPZIG

E-mail address: mugnai@mis.mpg.de, roeger@mis.mpg.de 